Article

\title{
Retrieval of a Temporal High-Resolution Leaf Area Index (LAI) by Combining MODIS LAI and ASTER Reflectance Data
}

\author{
Yonghua $Q u^{1,2,3, *}$, Wenchao Han ${ }^{1,2,3}$ and Mingguo Ma ${ }^{4}$
}

1 State Key Laboratory of Remote Sensing Science, Jointly Sponsored by Beijing Normal University and the Institute of Remote Sensing Applications of CAS, Beijing 100875, China; E-Mail: hwc9028@163.com

2 Beijing Key Laboratory for Remote Sensing of Environment and Digital Cities, Beijing Normal University, Beijing 100875, China

3 School of Geography, Beijing Normal University, Beijing 100875, China

4 Laboratory of Remote Sensing and Geospatial Science, Cold and Arid Regions Environmental and Engineering Research Institute of CAS, Lanzhou 730000, China;

E-Mail: mmg@1zb.ac.cn

* Author to whom correspondence should be addressed; E-Mail: qyh@bnu.edu.cn; Tel.: +86-10-5880-2041; Fax: +86-10-5880-7452.

Academic Editors: Xin Li, Yuei-An Liou, Qinhuo Liu and Prasad S. Thenkabail Received: 27 October 2014 / Accepted: 15 November 2014 / Published: 24 December 2014

\begin{abstract}
This paper aims to retrieve temporal high-resolution LAI derived by fusing MOD15 products (1 $\mathrm{km}$ resolution), field-measured LAI and ASTER reflectance (15-m resolution). Though the inversion of a physically based canopy reflectance model using high-resolution satellite data can produce high-resolution LAI products, the obstacle to producing temporal products is obvious due to the low temporal resolution of high resolution satellite data. A feasible method is to combine different source data, taking advantage of the spatial and temporal resolution of different sensors. In this paper, a high-resolution LAI retrieval method was implemented using a dynamic Bayesian network (DBN) inversion framework. MODIS LAI data with higher temporal resolution were used to fit the temporal background information, which is then updated by new, higher resolution data, herein ASTER data. The interactions between the different resolution data were analyzed from a Bayesian perspective. The proposed method was evaluated using a dataset collected in the HiWater (Heihe Watershed Allied Telemetry Experimental Research) experiment. The determination coefficient and RMSE between the estimated and measured LAI are 0.80 and
\end{abstract}


0.43 , respectively. The research results suggest that even though the coarse-resolution background information differs from the high-resolution satellite observations, a satisfactory estimation result for the temporal high-resolution LAI can be produced using the accumulated information from both the new observations and background information.

Keywords: leaf area index; dynamic Bayesian network; uncertainty analysis; high spatial resolution; high temporal resolution

\section{Introduction}

The leaf area index (LAI), defined as half of the leaf surface area on a unit ground surface area, is an important structural parameter of vegetation [1]. Retrieving LAIs from remotely sensed data is an efficient method compared to ground-based measurement [2]. Currently, several satellite LAI products have been available, such as MODIS [3], GEOV1 [4], GLASS [5], GLOBMAP [6], and JRC-TIP [7]. However, the above LAI products yield a coarse resolution from 500 to $3000 \mathrm{~m}$. In the context of land and resource monitoring in a small regional area, temporal high-resolution LAI products are more appreciated $[8,9]$.

One straightforward method to produce high resolution LAIs is using high resolution satellite data, such as Landsat TM/ETM+ [8] and ASTER VNIR [10,11] imagery with $30 \mathrm{~m}$ and $15 \mathrm{~m}$ resolution, respectively. However, the quantitative products from high-resolution remote-sensing observation data yield a discontinuous time series that suffers from bad weather conditions. Therefore, to obtain high-quality LAI products, researchers have begun to synthetically calculate LAIs using multi-source remote-sensing data using dynamic change information from existing remote-sensing products $[12,13]$.

On the one hand, when multi-source data are considered in producing high-resolution LAIs, more time information can be utilized to improve the quality, but on the other hand, certain aspects may yield uncertainties from the different source data (e.g., the mismatch between data resolution and inherent errors in an individual dataset). An uncertainty analysis on the estimated results will aid users in understanding how these products perform and improve the accuracy of future products [14]. From the perspective of understanding the interaction processes of information and improving model reliability, it is necessary to conduct uncertainty analysis on the inversion models $[15,16]$. Herein, the uncertainties about differently scaled data are analyzed using the posterior entropy of the temporal LAI estimation result.

This paper adopts a dynamic Bayesian network method to calculate temporal high-resolution LAI products by combining coarse resolution LAI dynamic information and higher resolution remotely sensed reflectance. The uncertainty analysis about the retrieved result is conducted on the posterior probability information that is derived from Bayesian inference, and the performance of the retrieved LAI was validated using ground measurement data. 


\section{Study Area and Data}

\subsection{Ground Experiment}

The study area is located in the Xiaoman irrigated district in the arid regions of the middle Heihe River, which is $8 \mathrm{~km}$ south of Zhangye in the Gansu province [17]. The study area is a typical farmland ecosystem. Its main type of vegetation is corn. We used a $4 \times 4 \mathrm{~km}^{2}$ range (which corresponds to $4 \times 4$ MODIS pixels) to arrange the ground experimental observations and collect the field LAI values. The ground experiment in the study area is part of the HiWATER (Heihe Watershed Allied Telemetry Experimental Research) experiment [17], which was conducted from June to August, 2012.

During the ground experimental period, the ground LAI of corn was measured using the wireless sensor network method LAINet [18]. The performance of LAINet has been widely validated through comparison with other commercial instruments, such as LAI-2000 [18] and TRAC [19]. Figure 1 shows the distribution of the 42 LAINet nodes (green symbols) in the study area. We collected the ground LAIs from 25 June to 24 August 2012.

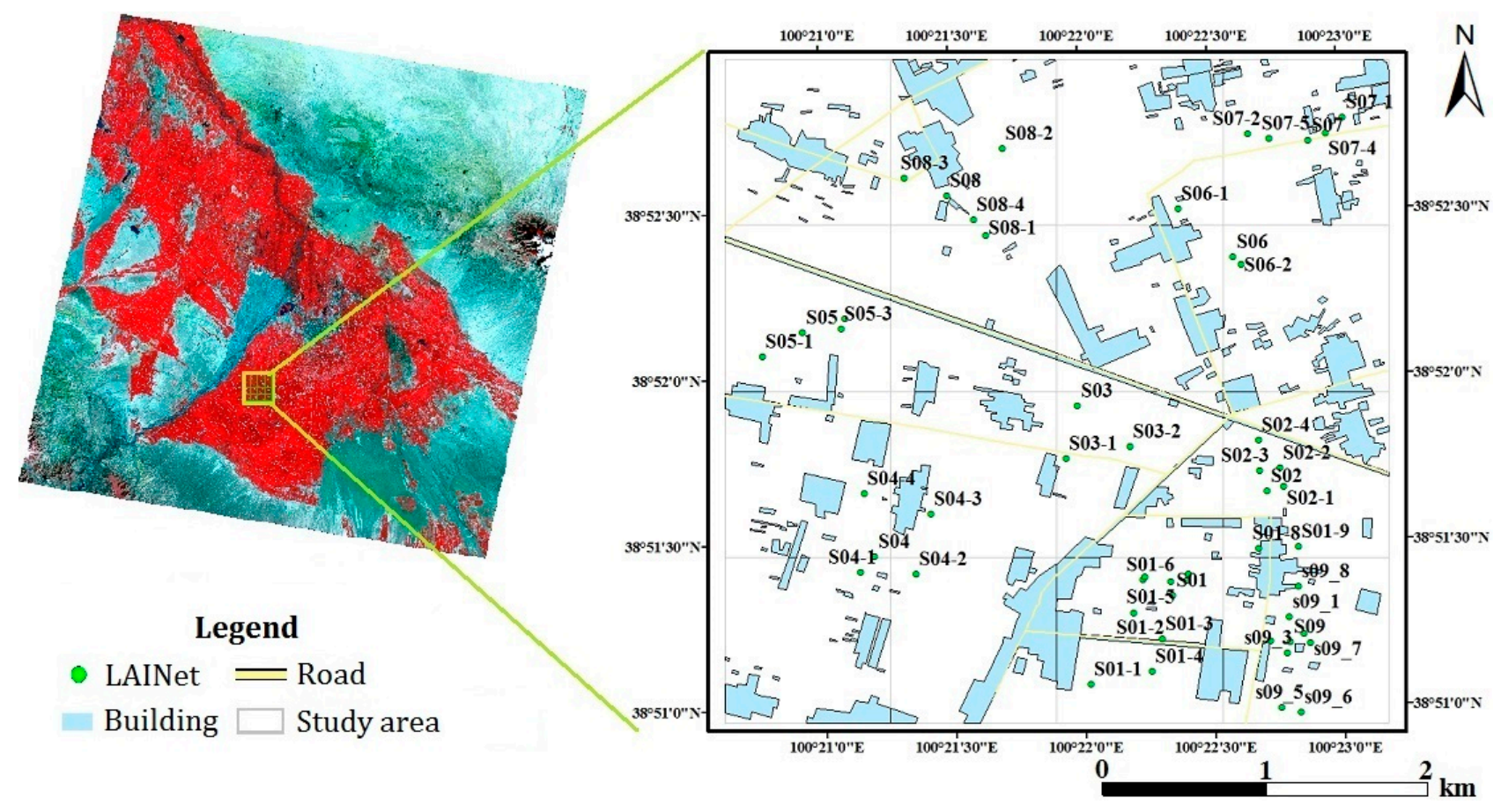

Figure 1. Distribution of the instruments in the study area. The green symbols are the location of the LAINet nodes.

\subsection{Data}

The data used in this article are MODIS LAI products (MOD15A2), ASTER images and the field measurements collected using LAINet, an automated LAI measurement system based on a wireless sensor network technique.

We used MODIS LAI products (MOD15A2) as background information to construct the LAI dynamic process. MOD15A2's spatial resolution and time interval were $1 \mathrm{~km}$ and eight days [3], respectively. MOD15A2's acquisition dates ranged from DOY (day of year) 153 to DOY 265 in 2012 
(which corresponded with the ground experiment time). Details on the above data are shown in Table 1. Before the MODIS LAI data can be used to establish the LAI dynamic process, the Savitzky-Golay filter [20] was used to reduce or eliminate the spatial and temporal discontinuity of the MODIS LAI time-series data.

Table 1. The spatial and temporal information of satellite data and the ground data.

\begin{tabular}{cccc}
\hline Data Source & Spatial Resolution & Temporal Resolution & DOY \\
\hline MOD15A2 & $1 \mathrm{~km}$ & 8 days & $153 / 161 / 169 / 177 / 185 / 193 / 201 / 209 / 217 / 225 / 233 / 241 / 249 / 257 / 265$ \\
ASTER & $15 \mathrm{~m}$ & Varied & $152 / 167 / 192 / 215 / 224 / 231 / 240 / 247 / 263$ \\
LAINet & ground point & 5 days & $177 \sim 232$ \\
\hline
\end{tabular}

The nine high-quality (high resolution, clearer, cloudless) ASTER surface reflectance images' data with 15-m resolution were used as high-resolution remote-sensing observations to update the background information. The specific times for the images are shown in Table 1. Before these data were used to invert the LAI, they were corrected through geometric calibration using a ground-measured settlements vector layer of the study area as the reference image, and the calibrated errors were within 0.5 pixels. MODIS is high temporal resolution, low spatial resolution whilst ASTER is high spatial resolution, low temporal resolution.

The field-measured LAINet data were used as ground truth data. We aggregated the observed daily LAINet data into a dataset with 5-days interval by calculating the average value of 5 days. The details on the processing method and results from a comparison with other instruments are in [18].

\section{Methods}

\subsection{Methods Overview}

The framework for estimating temporal, high-resolution LAIs is based on a dynamic Bayesian network (DBN), which combines the dynamic-change information from coarse-resolution LAI products with observation information from high-resolution remote-sensing data. In the DBN method, the dynamic-change information was introduced using a simple fitted equation, and the observation information was introduced through the canopy reflection model. The uncertainty of the multi-source information's interactions and estimated results were analyzed and validated using the field-measured data.

The diagrammatic chart for the proposed framework is shown in Figure 2 and is further divided into four steps. In the first step, the ASTER LAIs are generated using a constrained inversion method. Specifically, together with the ASTER reflectance data, some selected ground-truth values were used to generate the look up table (LUT), which was used to estimate the high-resolution LAIs. Several other methods also have been used: inversion of physically based canopy reflectance models using the traditional optimization method, neural networks, genetic algorithm [21]. The output from the first step includes 15-m-resolution ASTER LAIs and their corresponding posterior probability distributions. The process is shown in part A of Figure 2. In the second step, a fitted LAI growth equation was established using the MODIS LAI data to describe the background LAI information. The fitted equation was also used to calculate the LAI state transition probability between each time slice. The second step is shown 
in part B of Figure 2. In the third step, the information derived from the above steps is updated by the dynamic Bayesian network method. In this step, the state transition probability in part B is updated using the conditional probability distribution from part A; next, the information entropy and maximum probability were calculated using the updated posteriori probability distribution, which is shown in part $\mathrm{C}$ of Figure 2. Through an iteration composed of steps 1 to 3, the coarse-resolution background information is updated using high-resolution satellite observations, and the updated result is the LAI posteriori probability distribution for each time slice. All of the above steps will be introduced in detail in Section 3.2 to Section 3.5.

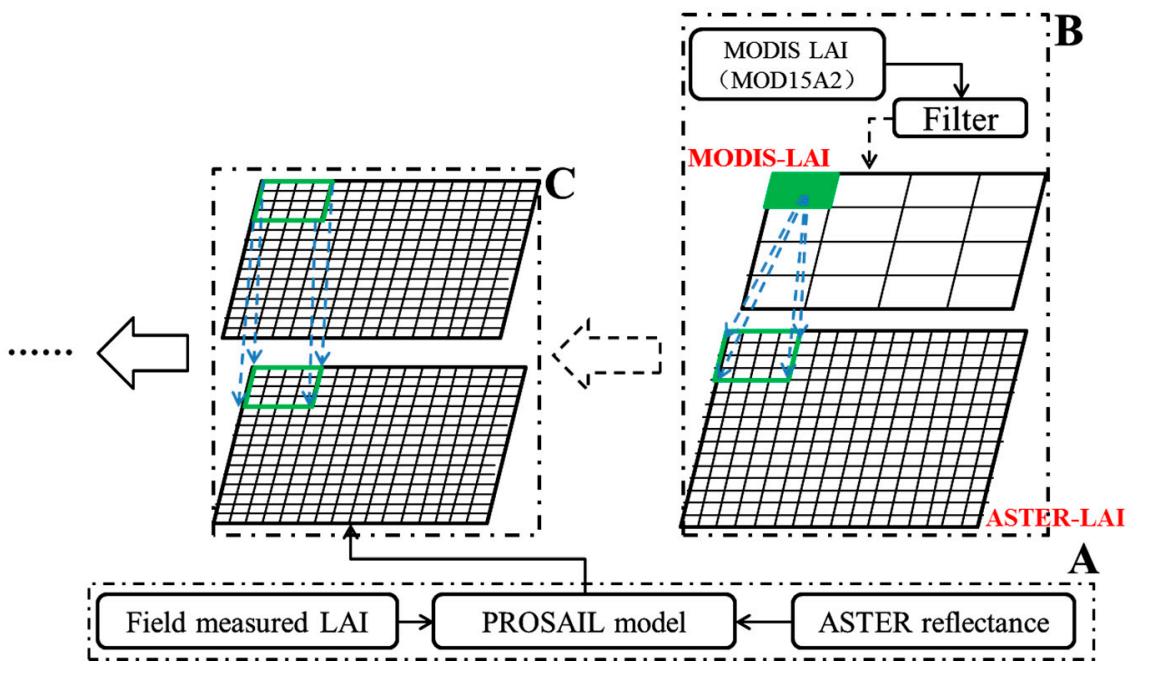

Figure 2. The diagrammatic chart for temporal, high-resolution leaf area index (LAI) retrieval.

\subsection{Step 1: Generating a Look-Up Table Constrained Using Ground Observations}

Currently, estimating the LAI from remote-sensing data will generally rely on a canopy reflectance (CR) model; however, a CR model has more than one free variable besides the target variable, so it is difficult to retrieve the value of the target variable by inverting the CR model. Many techniques have been developed to reduce the effects of the free variables and improve the reliability of the retrieved variable, such as using ground-truth data to constrain the model parameters [22-24]. In this work, there were 42 LAINet nodes deployed in the experiment area; as a result, many LAI data recorders were available and some of the data can be selected as constraints when inverting a CR model.

Here, a coupled radiation transfer model called PROSAIL (PROSPECT + SAIL) is used to generate the look-up table. The PROSAIL model consists of the leaf optical properties model PROSPECT [25] and the canopy reflectance model SAIL [26]. The PROSPECT model's input parameters involve chlorophyll content $\left(\mathrm{C}_{\mathrm{ab}}\right)$, dry matter content $\left(\mathrm{C}_{\mathrm{m}}\right)$, equivalent water thickness $\left[(\mathrm{EWT})-\mathrm{C}_{\mathrm{w}}\right]$ and the leaves' structural parameter $(\mathrm{N})$; the output of this model includes hemispherical reflectance and transmittance. The SAIL model's input parameters include leaf reflectance $\left(\rho_{L}\right)$ and leaf transmittance $\left(\tau_{L}\right)$, which were simulated using the PROSPECT model, the average leaf angle (ALA), soil reflectance $\left(\rho_{S}\right)$, horizontal visibility (VIS), view zenith angle $\left(\theta_{V}\right)$, solar zenith angle $\left(\theta_{S}\right)$, and relative azimuth angle $(\varnothing)$. The function relationship between the vegetation parameters as shown above and through the reflectance can be simply described as follows 


$$
\rho=\operatorname{PROSAIL}\left(C_{a b}, C_{m}, C_{w}, N, L A I, A L A, \rho_{s}, V I S, \theta_{v}, \theta_{s}, \phi\right)
$$

Before the ground data were used to constrain the inverted model, the sensitivity of the model's free variables was inspected. The specific process underlying this process can be further decomposed into three sub-processes. First, the ranges of the four PROSPECT input parameters were determined by the statistics from the LOPEX'93 dataset (Leaf Optical Properties Experiment) [27]. Second, we kept any three input parameters fixed while changing the remaining parameter within its statistical range, and its impact on the estimated LAI was documented. Third, the sensitivity of the four parameters was calculated and arranged from strong to weak. In this work, the calculated result is in the following order: $\mathrm{C}_{\mathrm{m}}, \mathrm{N}, \mathrm{C}_{\mathrm{ab}}$, and $\mathrm{C}_{\mathrm{w}}$. The sensitivity order reveals that the estimated LAI is least sensitive to $\mathrm{C}_{\mathrm{ab}}$ and $\mathrm{C}_{\mathrm{w}}$ (i.e., the LAI almost did not change when these parameters changed).

To implement Step 1, the ground-measured LAI values were selected using the criterion that there are pure vegetation pixels in the area surrounding the measured point within $45 \times 45 \mathrm{~m}^{2}$, which corresponds to ASTER $3 \times 3$ pixels. We used the four groups of measured data to fix the $\mathrm{C}_{\mathrm{m}}$ and $\mathrm{N}$ values. The soil reflectance can be calculated using the soil-reflectance (SOILSPECT) model [28]; the horizontal visibility (VIS) was set as a constant in accordance with the empirical experiment, and the solar and observation geometries can refer to the MODIS reflectance products. When all of the free variables have been determined, the LUT can be generated by forward simulating the PROSAIL model with varying LAI values. Consequently, the estimated LAI is the value that minimizes the deviation between the simulated and satellite reflectances. When the model and observed data uncertainties are considered, the estimated results are generally expressed as a probability distribution.

\subsection{Step 2: Fitting the LAI Growth Equation}

Unlike satellite observations, which directly drive the inverted canopy reflectance model, the dynamic change information of LAI growth is auxiliary information on the target parameter when the canopy reflectance model is inverted. There are many methods to describe the temporal trend for LAI growth, such as growth model simulation. However, compared with the complicated biophysical-based model, the statistical model, which is characterized by its simple form and capacity to drive data, is more easily used in application.

Here, we used a LAI growth equation fitting method using temporal MODIS LAIs developed by $\mathrm{Qu}$ [29]; thus, the fitted LAI growth-trend information is introduced into the inversion processes as background information. It should be noted that there is a scale mismatch between the background information and satellite observations; as a result, the trend for changes in one MODIS LAI pixel in the time series can only be regarded as a general trend for all of the ASTER pixels within $1 \times 1 \mathrm{~km}^{2}$. The formula is shown in Equation (2).

$$
P\left(L A I_{t+1}\right) \propto P\left(L A I_{t}\right) \times P\left(\frac{I_{t+1}}{I_{t}}\right)
$$

where $P(\cdot)$ indicates the probability distribution of the variables, so the uncertainties of different source data is conducted using probability operation; $l_{t+1}$ and $l_{t}$ are the filtered MODIS LAI values at the $t+1$ and $t$ time slices, and $I_{t+1} / I_{t}$ is the background information. $L A I_{t}$ and $L A I_{t+1}$ are the predicted ASTER 
LAIs in their corresponding time slices. The model is a simple dynamic iterative equation; the predicted value of the next moment $L A I_{t+1}$ is a function of the previous moment inversion value $L A I_{t}$.

As shown in part B of Figure 2, each pixel of a MODIS LAI has its own background information value because there is a spatial resolution difference, so each MODIS pixel corresponds to multiple ASTER pixels. In other words, there will be many ASTER pixels sharing the same background information. However, because every ASTER pixel has its own reflectance, after the background information is updated by high resolution observations, each ASTER pixel has its own $L A I_{t+1}$ and $L A I_{t}$ values. The uncertainties raised from different data scales are discussed in the following section.

\subsection{Step 3: Retrieving the Temporal High-Resolution LAI}

A method based on a dynamic Bayesian network was developed by Qu et al. [29,30] and is employed here to retrieve temporal high-resolution LAIs. We used reflectance and background information as preconditions to deduce the conditional probability of the LAIs, while the previous works $[29,30]$ used only reflectance data.

Figure 3 shows a diagram of the inversion process schematic for the dynamic Bayesian network. $A_{R E F \sim T}$ is the high-resolution remote-sensing observation at time T. $M_{L A I \sim T}$ is the MODIS LAI, and $A_{L A I \sim T}$ is the inversion result at time T; the arrow indicates the relationship between the parameters. The green rectangular box indicates the growth model prediction based on the background information, and the red rectangle box indicates the inversion process restrained by the high-resolution observation and field-measured data. In the proposed method, which takes advantage of the fitted model predication, not every moment throughout the time series needs remote-sensing observation data.

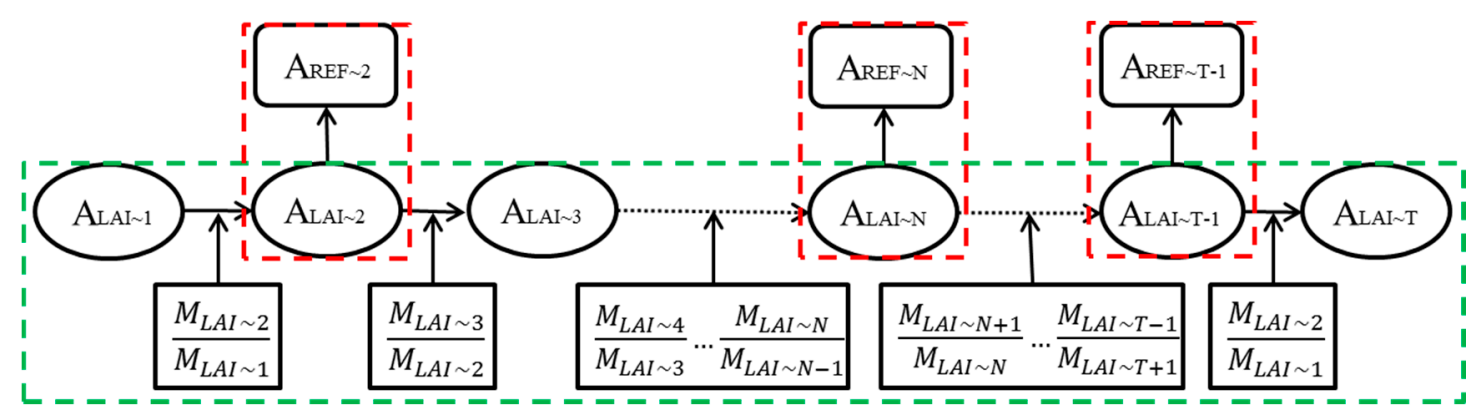

Figure 3. The schematic diagram for dynamic Bayesian network retrieval.

Assuming that the high-resolution remote-sensing observation data $\left(A_{R E F \sim 1}, A_{R E F \sim N}, \cdots, A_{R E F \sim T}\right)$ and MODIS LAIs with eight-day intervals $\left(M_{L A I \sim 1}, M_{L A I \sim 2}, M_{L A I \sim 3}, \cdots, M_{L A I \sim T-1}, M_{L A I \sim T}\right)$ are available, the posterior probability of $A_{L A I \sim T}$ at time T, which is denoted as $P\left(A_{L A I \sim T} \mid A_{R E F \sim T}, M_{L A I \sim T}\right)$, can thus be derived using the Bayes theorem:

$$
P\left(A_{L A I^{\sim} T} \mid A_{R E F^{\sim} T}, M_{L A I^{\sim} T}\right)=\frac{P\left(A_{R E F^{\sim} T} \mid A_{L A I^{\sim} T}\right) \times \sum_{T-1=k} P\left(A_{L A I^{\sim} T} \mid A_{L A I^{\sim} T-1}\right) \times P\left(A_{L A I^{\sim} T-1} \mid M_{L A I^{\sim} T-1}\right)}{P\left(A_{R^{\sim} \sim T} \mid M_{L A I^{\sim} T}\right)}
$$

This formula is implemented through the following three steps. (1) The canopy reflection model is used to calculate the conditional probability at time $T, P\left(A_{R E F \sim T} \mid A_{L A I \sim T}\right)$; (2) The dynamic fitted model 
is used to calculate the state transition probability at time $T, P\left(A_{L A I \sim T} \mid A_{L A I \sim T-1}\right)$; (3) The probability distribution of (1) is used to update the probability distribution of (2) and obtain the posteriori probability of the LAI at time $T$. When a high-resolution remote-sensing observation is available, if the probabilities of (1) and (2) have been calculated, the posterior probability distribution of the LAI at this moment can be calculated.

To estimate LAIs based on the posterior probability distribution of each moment, two methods are available. One requires taking the value that corresponds to the maximum probability of the posterior probability distribution as the final LAI; the other requires taking the expectation of the posterior probability distribution as the final LAI. Here, we used the first method to calculate the LAI for each moment.

\subsection{Step 4: Calculating the Information Entropy and Maximum Probability}

The information entropy represents the statistical characteristics of the information source, and it can measure the randomness of the information source and average uncertainty of the probability distribution. Through calculating the information entropy of the LAI probability distribution, we can analyze the uncertainty for the LAI of each pixel for each time slice. The formula for calculating information entropy is shown in Equation (4) [31]:

$$
H\left(x_{i}\right)=E\left[\log _{2} 1 / p\left(x_{i}\right)\right]=\sum p\left(x_{i}\right) \log _{2}\left(p\left(x_{i}\right)\right)
$$

$H\left(x_{i}\right)$ is the information entropy, and $p\left(x_{i}\right)$ is the probability value in the probability distribution. In the Bayesian inference process, each LAI inversion result is expressed through a posterior probability distribution, which can be used to calculate its information entropy. A higher information entropy value represents a greater uncertainty; in contrast, a lower information entropy value represents less uncertainty.

As addressed in Section 3.4, the estimated LAI is determined by the value with the maximum probability in the estimated posterior probability distribution. As a result, when the LAI value is retrieved, it also has a corresponding probability value. A higher maximum probability value represents greater LAI reliability; in contrast, a smaller maximum probability value represents lower LAI reliability.

In evaluating the uncertainty of the estimated results, the results are acceptable if the shape of the LAI posteriori probability distribution is more concentrated, its maximum probability value is higher, and its information entropy is smaller; under these conditions, the LAI reliability is greater. Thus, calculating the information entropy and maximum probability of the posterior probability distribution can provide a quantitative analysis and evaluation for the reliability of the LAI inversion results (i.e., the uncertainty).

\section{Results and Discussion}

\subsection{PROSAIL Model Parameters Determined through Fixed Ground Measurements}

Four ground point values were selected from three days of measurement data as constraints to determine the PROSAIL model's parameters. The information for these data is shown in Table 2. Using the method illustrated in Section 3.2, the PROSAIL model's parameter values (or ranges) used to create the LUT were determined; the results are in Table 3. 
Table 2. Information on the measured points used to constrain the input parameters.

\begin{tabular}{cccc}
\hline ID & DOY & Lon & Lat \\
\hline 258 & $192,207,222$ & $100.36976 \mathrm{E}$ & $38.854710 \mathrm{~N}$ \\
259 & $192,207,222$ & $100.35221 \mathrm{E}$ & $38.856950 \mathrm{~N}$ \\
286 & $192,207,222$ & $100.35146 \mathrm{E}$ & $38.869710 \mathrm{~N}$ \\
229 & $192,207,222$ & $100.34615 \mathrm{E}$ & $38.867900 \mathrm{~N}$ \\
\hline
\end{tabular}

Table 3. The PROSAIL input parameters.

\begin{tabular}{cccccc}
\hline Variables & Unit & Range & Variables & Unit & Range \\
\hline $\mathrm{C}_{\mathrm{ab}}$ & $\mu \mathrm{g} / \mathrm{cm}^{2}$ & 30 & $\rho_{S}$ & - & 0.232 \\
$\mathrm{C}_{\mathrm{m}}$ & $\mathrm{g} / \mathrm{cm}^{2}$ & 0.008 & $\mathrm{VIS}$ & $\mathrm{km}$ & 20 \\
$\mathrm{C}_{\mathrm{w}}$ & $\mathrm{cm}$ & 0.0191 & $\theta_{V}$ & degree & $20 \sim 30$ \\
$\mathrm{~N}$ & - & 1.518 & $\theta_{S}$ & degree & $35 \sim 45$ \\
$\mathrm{LAI}$ & $\mathrm{m}^{2} / \mathrm{m}^{2}$ & $0 \sim 6.5$ & $\varnothing$ & degree & 125 \\
$\mathrm{ALA}$ & degree & 45 & & & \\
\hline
\end{tabular}

\subsection{Evaluation of the Performance of the Estimated LAI}

Figure 4 is the scatter plot for the estimated LAI and field-measured values (30 field-measured points were selected). In this figure, the four groups of field-measured data that were previously used to constrain the PROSAIL model's parameters were not included, and the remaining data were used to verify the inversion results. Figure 4 shows that the determination coefficient $\mathrm{R}^{2}$ and RMSE between the estimated and measured LAI were 0.80 and 0.43 , respectively. It is suggested that the inversion results are highly credible in general.

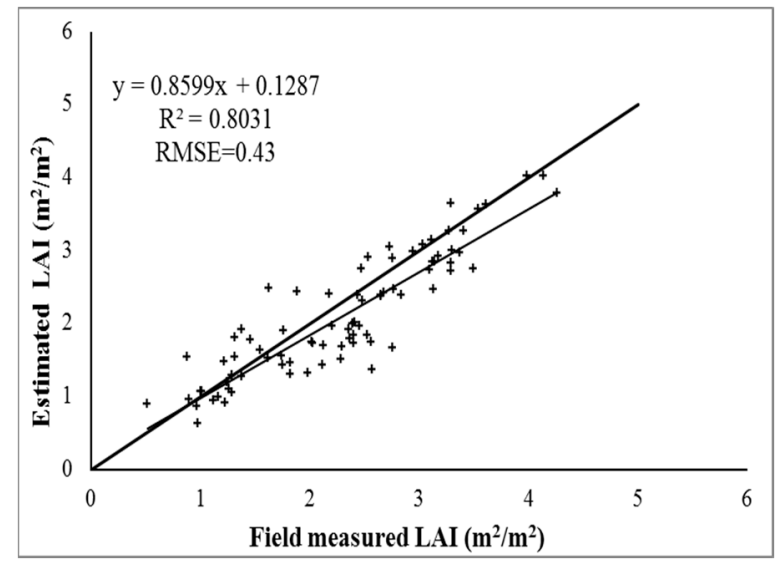

Figure 4. The scatter plot figure with the estimated and field-measured values.

\subsection{The Time-Series Analysis of the Estimated LAI}

High-spatial-resolution LAI images of corn throughout the growing season in the study area were obtained using the dynamic Bayesian network. The ASTER inversion results for 30 May 2012 were selected as the initial values for the dynamic process model. The time interval is $6 \sim 8$ days (because the times for the ASTER and MODIS deviate by 1 2 days, the time interval for the inversion results is not fixed at eight days), the time ranges from 1 June to 21 September 2012, and the spatial resolution is $15 \mathrm{~m}$; 
images of the results are shown in Figure 5. The images with dates underlined in Figure 5 represent highresolution remote-sensing observations that were used to update background information at the indicated date, and their results simultaneously contain the dynamic change information for the MODIS LAIs and the spatial information of the high-resolution remote-sensing observations. The remaining images without underlined dates represent moments without high-resolution remote-sensing observations, the results for which only contain dynamic change information.

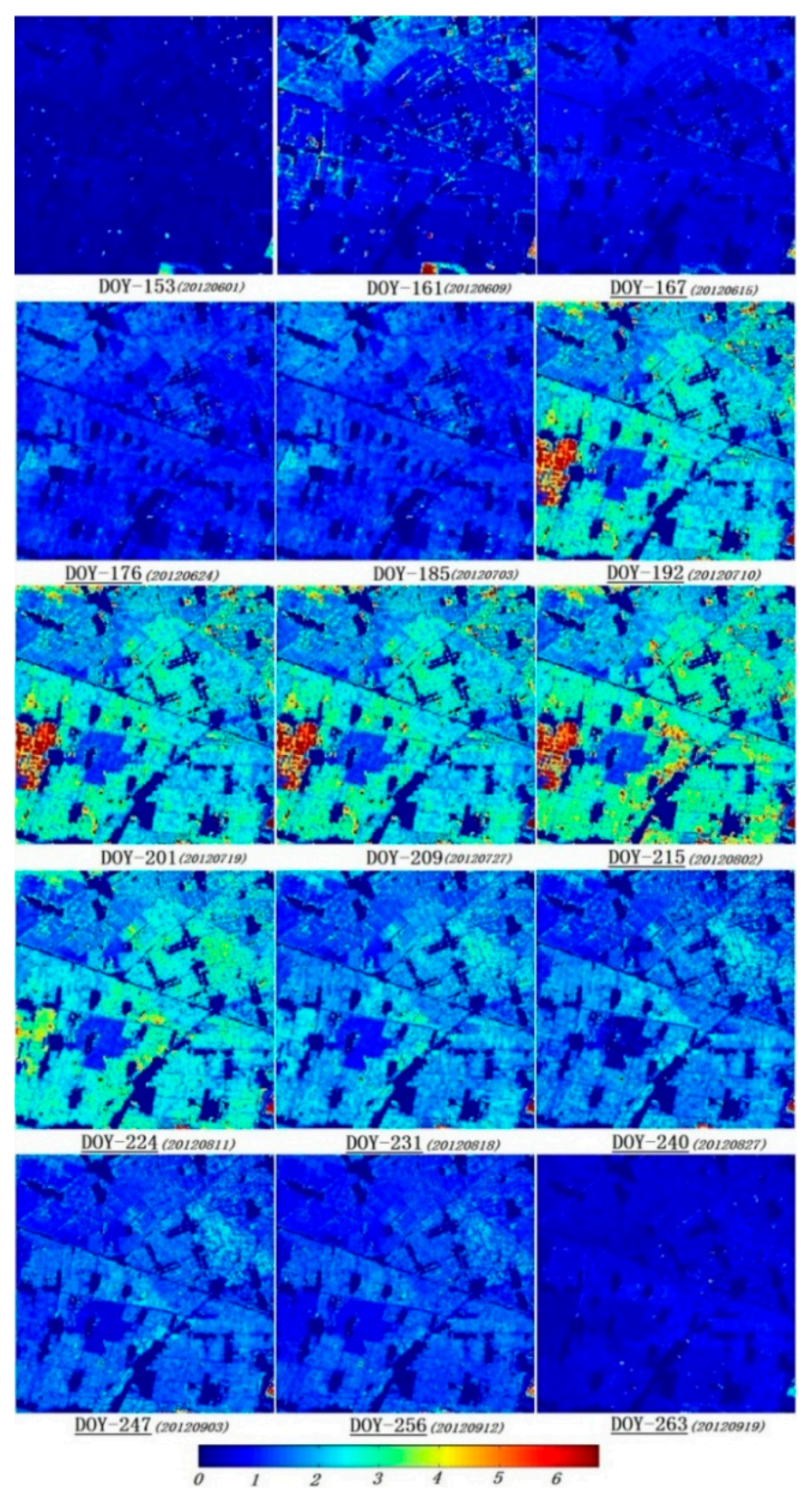

Figure 5. The high-resolution and time-series results for the estimated LAIs from the ASTER and MODIS LAI data for the year 2012; the underlined data were estimated from the ASTER and MODIS LAI data.

Figure 5 shows that at the beginning of the inversion, DOY-153 DOY-161, the inversion results are mainly influenced by the dynamic change information from the MODIS LAI because the high-resolution remote-sensing observations were not used to update the background information. The different MODIS 
LAI pixels exhibit different dynamic change characteristics, which clearly exhibit stripes between the pixels on the 161st day. As mentioned in Section 3.3, the dynamic change information errors produced by the scale differences produce errors in the results. However, after the 167 th day, with high-resolution remote-sensing observations gradually incorporated into the model through the time series, our inversion model can correct the deviations in the previous moment and continuously increase the inversion result's accuracy.

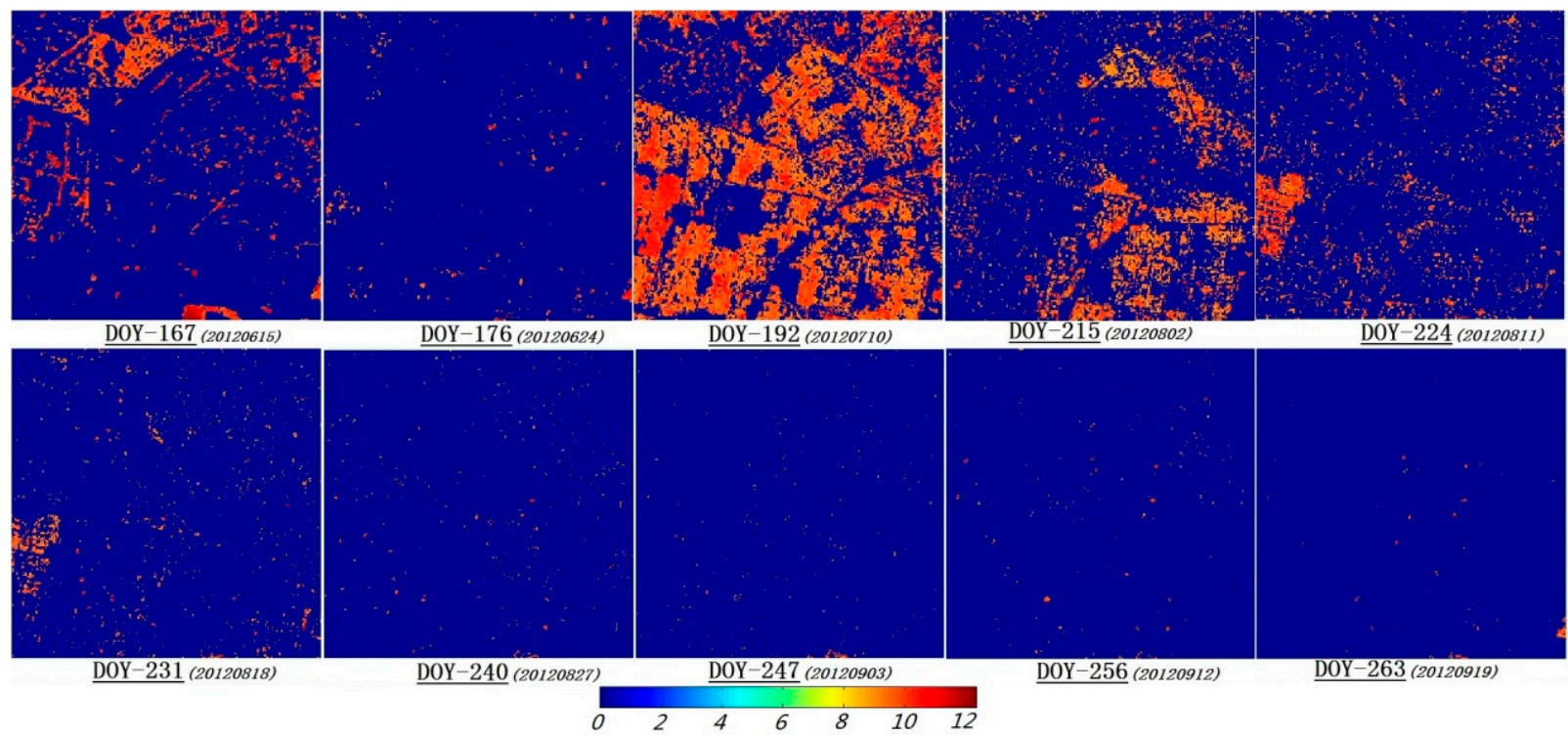

(a)
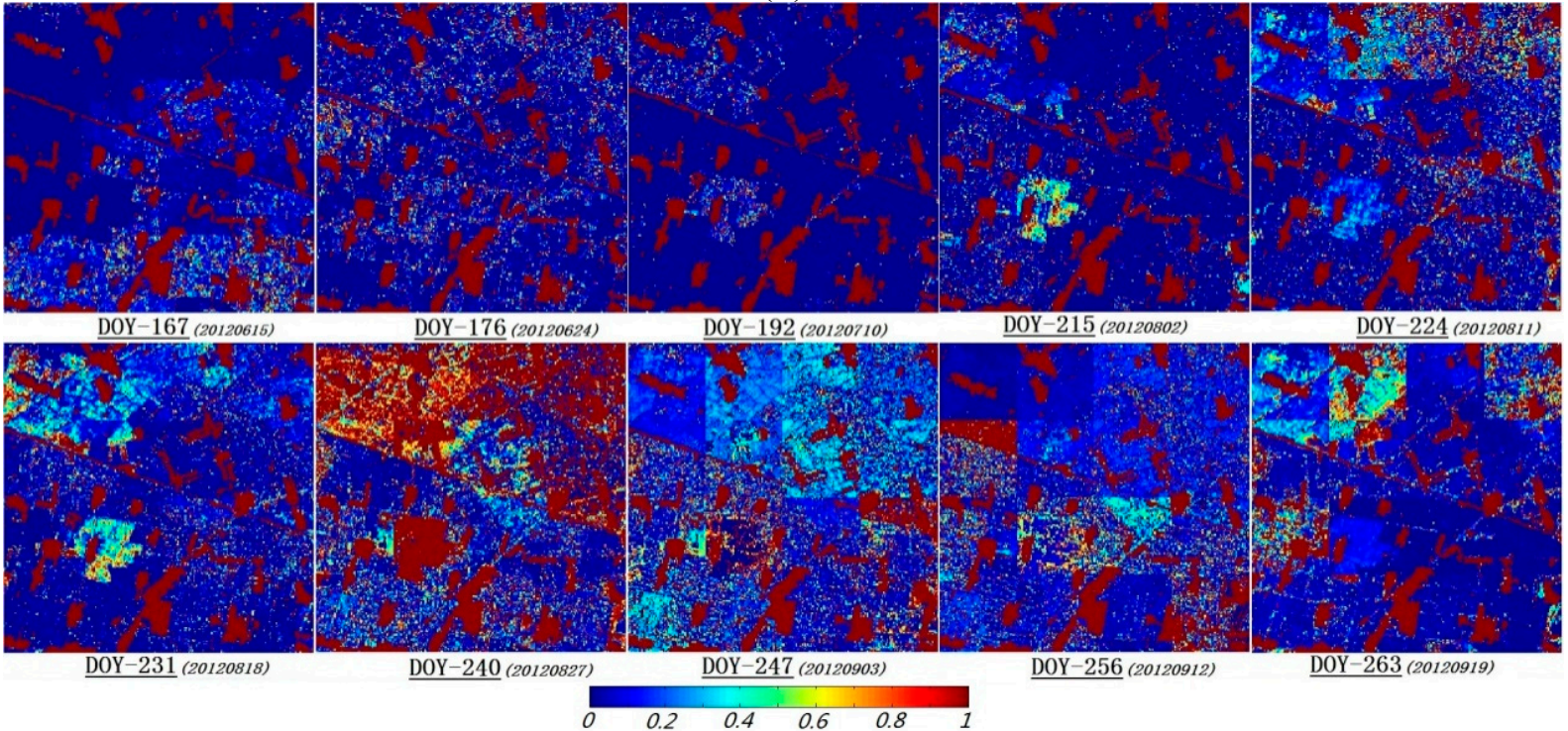

(b)

Figure 6. The updated high-resolution remote-sensing observation results for the information entropy and maximum probability; (a) information entropy, (b) maximum probability. 


\subsection{The Uncertainty Analysis for the LAI Inversion}

Figure 6 shows the information entropy and maximum probability results, which correspond to the time when the high-resolution remote-sensing observations were available. Figure 6a shows the information entropy results, and Figure $6 \mathrm{~b}$ shows the maximum probability results. In Figure 6a,b, obvious stripes appear (such as on the 167th day) on the initial day, mainly because each MODIS LAI was initially derived from different fitted dynamic process models. Therefore, larger deviations between the values predicted by the fitted model and inverted by the PROSAIL model will produce a lower maximum probability and higher information entropy, and vice versa. Therefore, if the higher resolution information is insufficient for injection into the inversion process, the estimated results can only reflect the coarser resolution information.

To further investigate the information and uncertainty variation throughout the study area using a time series, we re-scaled the calculated information entropy into a range from 0 to 1 . The re-scaled value and the mean and standard deviation of the information entropy and maximum probability were plotted in Figure 6. The results show that the variation tendency for the information entropy and maximum probability are opposed. Although the maximum probability of the 247 th day exhibited a downward trend, it remained in a high value range compared with the previous values. Similarly, the time to the information entropy peak was consistent with the time to the lowest maximum probability, which is consistent with their meaning; the maximum probability represents the highest probability for a certain value, and a lower maximum probability corresponds to a greater uncertainty. The information entropy represents the degree of information chaos; higher information entropy corresponds to greater uncertainty. Thus, the information entropy negatively correlates with the maximum probability.

Through calculating the information entropy and maximum probability of the posterior probability, where high-resolution remote-sensing observation data are gradually incorporated, the information entropy for the LAI posterior probability presents a decreasing trend, and the maximum probability presents an increasing trend. Thus, if the posterior information for the LAI gradually accumulates, the LAI reliability continually increases.

\section{Conclusions}

To retrieve the temporal high-resolution LAI products, this paper proposed a method to combine three sources of data from the Bayesian probability framework, in which the dynamic-change information coming from fitted coarse-resolution LAI products were used as the temporal background information and both high-resolution remote-sensing observation data and field-measured LAI were used as the new observed information. The dynamic Bayesian network was then used to combine the canopy reflectance model and fitted dynamic model. Moreover, the information entropy and maximum probability calculated from the probability distribution were used to analyze the uncertainties of the retrieved results. Based on the results, the following conclusions can be made:

(1) The determination coefficient $\mathrm{R}^{2}$ and RMSE between the estimated and measured LAIs are 0.80 and 0.43 , respectively. Thus, using multisource data to invert time-series and high-resolution LAIs is feasible, and it is an effective method to solve the problems with current remote sensing products, for which the resolution is low and the time is discontinuous. 
(2) The quantity of high-resolution remote-sensing observation data is an important factor for the inversion accuracy throughout the time series. In this paper, nine images of high-resolution remote-sensing observation data were used to update the background information, which constitutes nearly two-thirds of the inversion results. However, when the data are rarely updated, depending solely on the model's prediction may lead to predicted values that gradually deviate from the real values; this deviation cannot be corrected until the observation data are provided.

(3) Calculating the information entropy and maximum probability of the probability distribution not only quantitatively expresses the uncertainties of various states in the inversion process but also provides the change in the uncertainty during the information interaction process. The results demonstrate that in most cases, high-resolution observation data can provide effective information to update the background information. Thus, this method is feasible for inverting temporal high-resolution LAIs by incorporating the coarse-resolution LAI products and high-resolution remote-sensing observation data.

The current results also suggest that other aspects should be considered for future work. First, the background information data's quality should be taken into account. MOD15A2 is acquired from a single sensor, the data quality for which is affected by weather conditions, such as cloud cover and aerosols. Thus, to improve the reliability of the background information, MCD15A2, which is acquired from two synthesized sensors, may be an alternative that could be employed in future work. Second, the posterior information can be used to release more information. Furthermore, the PROSAIL model is selected to simulate the crop canopy reflectance in our current work; if possible, when the forest vegetation parameters are conducted, the geometric-optical model, such as Li-Strahler model [32,33] should be taken into consideration. Finally, in the current work, the coarse-resolution $(1 \mathrm{~km})$ LAI products were used as background information to invert high-resolution $(15 \mathrm{~m})$ LAI products. However, from the perspective of information iteration, the retrieved posterior information can thus be further used as background information if higher-resolution remote-sensing data (such as Quickbird or Worldview data) are available to invert the higher resolution LAI products.

\section{Acknowledgments}

This work was supported in part by the National Natural Science Foundation of China (41271348), the National Basic Research Program of China (2013CB733403), and in part by the National High Technology Research and Development Program (2012AA12A303) and the project as HIWATER: calibration and validation (91125004).

\section{Author Contributions}

Yonghua $\mathrm{Qu}$ designed the framework of this research work, and finished the final version of this paper. Wenchao Han processed the ASTER data and drafted the preliminary version of this paper. Mingguo Ma collected and preprocessed the field data.

\section{Conflicts of Interest}

The authors declare no conflict of interest. 


\section{References}

1. Chen, J.M.; Black, T.A. Defining leaf area index for non-flat leaves. Plant Cell Environ. 1992, 15, 421-429.

2. Fernandes, R.; Miller, J.R.; Hu, B.; Rubinstein, I.G. A multi-scale approach to mapping effective leaf area index in boreal picea mariana stands using high spatial resolution CASI imagery. Int. J. Remote Sens. 2002, 23, 3547-3568.

3. Myneni, R.; Hoffman, S.; Knyazikhin, Y.; Privette, J.; Glassy, J.; Tian, Y.; Wang, Y.; Song, X.; Zhang, Y.; Smith, G. Global products of vegetation leaf area and fraction absorbed PAR from year one of MODIS data. Remote Sens. Environ. 2002, 83, 214-231.

4. Camacho, F.; Cernicharo, J.; Lacaze, R.; Baret, F.; Weiss, M. Geov1: LAI, FAPAR essential climate variables and fcover global time series capitalizing over existing products. Part 2: Validation and intercomparison with reference products. Remote Sens. Environ. 2013, 137, 310-329.

5. Xiao, Z.; Liang, S.; Wang, J.; Chen, P.; Yin, X.; Zhang, L.; Song, J. Use of general regression neural networks for generating the glass leaf area index product from time-series MODIS surface reflectance. IEEE Trans. Geosci. Remote Sens. 2014, 52, 209-223.

6. Liu, Y.; Liu, R.; Chen, J.M. Retrospective retrieval of long-term consistent global leaf area index (1981-2011) from combined AVHRR and MODIS data. J. Geophys. Res.: Biogeosci. 2012, doi:10.1029/2012JG002084.

7. Pinty, B.; Andredakis, I.; Clerici, M.; Kaminski, T.; Taberner, M.; Verstraete, M.; Gobron, N.; Plummer, S.; Widlowski, J.L. Exploiting the MODIS albedos with the two-stream inversion package (JRC-TIP): 1. Effective leaf area index, vegetation, and soil properties. J. Geophys. Res.: Atmos. 2011, doi:10.1029/2010JD015372.

8. Gonzalez-Sanpedro, M.C.; Le Toan, T.; Moreno, J.; Kergoat, L.; Rubio, E. Seasonal variations of leaf area index of agricultural fields retrieved from landsat data. Remote Sens. Environ. 2008, 112, 810-824.

9. Colomboa, R.; Bellingerib, D.; Fasolinic, D.; Marinob, C.M. Retrieval of leaf area index in different vegetation types using high resolution satellite data. Remote Sens. Environ. 2003, 86, 120-131.

10. Jiménez-Muñoz, J.C.; Sobrino, J.A.; Gillespie, A.; Sabol, D.; Gustafson, W.T. Improved land surface emissivities over agricultural areas using ASTER NDVI. Remote Sens. Environ. 2006, 103, 474- 487.

11. Fernández-Manso, O.; Fernández-Manso, A.; Quintano, C. Estimation of aboveground biomass in mediterranean forests by statistical modelling of ASTER fraction images. Int. J. Appl. Earth Observ. Geoinf. 2014, 31, 45-56.

12. Gray, J.; Song, C. Mapping leaf area index using spatial, spectral, and temporal information from multiple sensors. Remote Sens. Environ. 2012, 119, 173-183.

13. Gao, F.; Anderson, M.C.; Kustas, W.P.; Wang, Y. Simple method for retrieving leaf area index from landsat using MODIS leaf area index products as reference. J. Appl. Remote Sens. 2012, 6, 063554-063551.

14. Fang, H.; Jiang, C.; Li, W.; Wei, S.; Baret, F.; Chen, J.M.; Garcia-Haro, J.; Liang, S.; Liu, R.; Myneni, R.B. Characterization and intercomparison of global moderate resolution leaf area index (LAI) products: Analysis of climatologies and theoretical uncertainties. J. Geophys. Res.: Biogeosci. 2013, $118,529-548$. 
15. Aggarwal, P. Uncertainties in crop, soil and weather inputs used in growth models: Implications for simulated outputs and their applications. Agric. Syst. 1995, 48, 361-384.

16. Wulder, M.A.; White, J.C.; Nelson, R.F.; Næsset, E.; Ørka, H.O.; Coops, N.C.; Hilker, T.; Bater, C.W.; Gobakken, T. Lidar sampling for large-area forest characterization: A review. Remote Sens. Environ. 2012, 121, 196-209.

17. Li, X.; Cheng, G.; Liu, S.; Xiao, Q.; Ma, M.; Jin, R.; Che, T.; Liu, Q.; Wang, W.; Qi, Y. Heihe watershed allied telemetry experimental research (HiWATER): Scientific objectives and experimental design. Bull. Am. Meteorol. Soc. 2013, 94, 1145-1160.

18. Qu, Y.; Zhu, Y.; Han, W.; Wang, J.; Ma, M. Crop leaf area index observations with a wireless sensor network and its potential for validating remote sensing products. IEEE J. Sel. Top. Appl. Earth Observ. Remote Sens. 2014, 7, 431-444.

19. Qu, Y.; Han, W.; Fu, L.; Li, C.; Song, J.; Zhou, H.; Bo, Y.; Wang, J. LAINET-A wireless sensor network for coniferous forest leaf area index measurement: Design, algorithm and validation. Comput. Electron. Agr. 2014, 108, 200-208.

20. Press, W.H.; Teukolsky, S.A. Savitzky-golay smoothing filters. Comput. Phys. 1990, 4, 669-672.

21. Fang, H.; Liang, S.; Kuusk, A. Retrieving leaf area index using a genetic algorithm with a canopy radiative transfer model. Remote Sens. Environ. 2003, 85, 257-270.

22. Tian, Y.; Woodcock, C.E.; Wang, Y.; Privette, J.L.; Shabanov, N.V.; Zhou, L.; Zhang, Y.; Buermann, W.; Dong, J.; Veikkanen, B. Multiscale analysis and validation of the MODIS LAI product: I. Uncertainty assessment. Remote Sens. Environ. 2002, 83, 414-430.

23. Wang, T.; Lü, C.; Yu, B. Assessing the potential productivity of winter wheat using wofost in the Beijing-Tianjin-Hebei region. J. Nat. Resour. 2010, 25, 475-487.

24. Zhu, X.; Zhao, Y.; Feng, X. A methodology for estimating leaf area index by assimilating remote sensing data into crop model based on temporal and spatial knowledge. Chin. Geogr. Sci. 2013, 23, $550-561$.

25. Jacquemoud, S.; Baret, F. Prospect: A model of leaf optical properties spectra. Remote Sens. Environ. 1990, 34, 75-91.

26. Verhoef, W. Light scattering by leaf layers with application to canopy reflectance modeling: The sail model. Remote Sens. Environ. 1984, 16, 125-141.

27. Hosgood, B.; Jacquemoud, S.; Andreoli, G.; Verdebout, J.; Pedrini, G.; Schmuck, G. Leaf Optical Properties EXperiment 93 (LOPEX93); Report EUR-16095-EN; European Commission, Joint Research Centre, Institute for Remote Sensing Applications: Ispra, Italy, 1995.

28. Jacquemoud, S.; Baret, F.; Hanocq, J.F. Modeling spectral and bidirectional soil reflectance. Remote Sens. Environ. 1992, 41, 123-132.

29. Qu, Y.; Zhang, Y.; Wang, J. A dynamic bayesian network data fusion algorithm for estimating leaf area index using time-series data from in situ measurement to remote sensing observations. Int. J. Remote Sens. 2012, 33, 1106-1125.

30. Zhang, Y.; Qu, Y.; Wang, J.; Liang, S.; Liu, Y. Estimating leaf area index from MODIS and surface meteorological data using a dynamic bayesian network. Remote Sens. Environ. 2012, 127, 30-43.

31. Shannon, C.E. A mathematical theory of communication. Bell Syst. Techn. J. 1948, 27, 379-423, 623-656. 
32. Li, X.; Strahler, A.H. Geometric-optical modeling of a conifer forest canopy. IEEE Trans. Geosci. Remote Sens. 1985, GE-23, 705-721.

33. Wanner, W.; Li, X.; Strahler, A. On the derivation of kernels for kernel-driven models of bidirectional reflectance. J. Geophys. Res.: Atmos. 1995, 100, 21077-21089.

(C) 2014 by the authors; licensee MDPI, Basel, Switzerland. This article is an open access article distributed under the terms and conditions of the Creative Commons Attribution license (http://creativecommons.org/licenses/by/4.0/). 